\title{
A systematic review comparing neurodevelopmental outcome in term infants with hypoxic and vascular brain injury with and without seizures
}

\author{
T. R. De Haan ${ }^{1 *}$, J. Langeslag ${ }^{1}$, J. H. van der Lee ${ }^{2}$ and A. H. van Kaam ${ }^{1}$
}

\begin{abstract}
Background: There is increasing evidence that neonatal seizures in term neonates with stroke, asphyxia or brain haemorrhage might be associated with adverse neurodevelopment and development of epilepsy. The extent of this association is not known. The objective of this study was to assess the possible impact of neonatal seizures on these outcomes and if possible calculate a relative risk.

Methods: A systematic review and meta-analysis was performed (study period January 2000-June 2015). PubMed, Medline and Embase were searched for cohort studies evaluating neurodevelopmental outcome at the age of at least 18 months or development of epilepsy in surviving term neonates with or without neonatal seizures. The methodological quality of included studies was assessed and data extractions were performed in a standardized manner by independent reviewers. Pooled Relative Risks (RR) with 95\% confidence intervals for adverse outcome were calculated if possible.

Results: Out of 1443 eligible studies 48 were selected for full text reading leaving 9 cohort studies for the final analyses (4 studies on stroke, 4 on perinatal asphyxia and one on cerebral hemorrhage). For all cases with stroke or asphyxia combined the pooled risk ratio (RR) for adverse outcome when suffering neonatal seizures was 7.42 (3.8414.34); for neonates with perinatal asphyxia: 8.41 (4.07-17.39) and for neonates with stroke: 4.95 (1.07-23.0). The pooled RR for development of late onset epilepsy could only be determined for infants suffering from stroke: 1.48 (0.82-2.68). Results were biased and evidence sparse.

Conclusions: The presence of neonatal seizures in term newborns with vascular or hypoxic brain injury may have an impact on or be a predictor of neurodevelopmental outcome. The biased available data yield insufficient evidence about the true size of this association.
\end{abstract}

\section{Background}

Neonatal seizures are frequently encountered with a reported yearly incidence of $1-3$ per 1000 live births [1]. Neonatal seizures confront clinicians with many diagnostic and therapeutic challenges in the neonatal intensive care unit (NICU) [2]. The developing brain is highly vulnerable to hypoxic-ischemic insults and these insults therefore often result in neonatal seizures $[3,4]$.

\footnotetext{
* Correspondence: t.r.dehaan@amc.uva.nl

${ }^{1}$ Department of Neonatology, Emma Children's Hospital, Academic Medical

Center, PO Box 22660, 1100, DD, Amsterdam, the Netherlands

Full list of author information is available at the end of the article
}

It has been hypothesized that this hyper excitability of the brain is caused by interplay between a high density synaptic network, an abundance of glutaminergic neurons, paradoxical neonatal excitatory actions of primary inhibitory networks and differences in membrane potentials in the immature dendrites $[1,3,5]$.

The most important causes of neonatal seizures are hypoxic ischemic encephalopathy (HIE) due to perinatal asphyxia (50-60\%), stroke (10\%), and cerebral haemorrhage $(10 \%)[3,6]$.

There is increasing evidence that neonatal seizures are associated with adverse neurodevelopmental outcome, defined as cerebral palsy, psychomotor retardation and

(c) The Author(s). 2018 Open Access This article is distributed under the terms of the Creative Commons Attribution 4.0 International License (http://creativecommons.org/licenses/by/4.0/), which permits unrestricted use, distribution, and 
the development of post-neonatal epilepsy [2, 4, 7-9]. This may be caused by the fact that neonatal seizures add damage to an already injured brain $[3,10]$ and to the fact that neonatal seizures alter neuronal circuitry in the developing brain leading to an enhanced susceptibility to post-neonatal epilepsy [1].

This study's objective was to determine if the presence of seizures in newborns with a perinatal insult to the brain (either hypoxia-asphyxia, stroke or hemorrhage) is associated with a worse long term outcome and increased risk for epilepsy in survivors. To answer this question a systematic review on this topic, including a meta-analysis were performed.

\section{Methods}

We followed the guidelines from the Meta-analysis of Observational Studies in Epidemiology (MOOSE) [11], Preferred Reporting Items for Systematic Reviews and Meta-Analyses the (PRISMA) statements [12], and the Cochrane Handbook for Systematic Reviews of Diagnostic Test Accuracy. For this study none of the authors had any competing interests.

\section{Search strategy}

We searched the bibliographical databases PubMed, Medline and Embase for cohort studies evaluating the long term neurodevelopmental outcome and later development of epilepsy in term newborns with hypoxic or vascular brain injury suffering from neonatal seizures.

The study period ranged from January 2000 - up until June 2015 and the search syntax can be found in Additional file 1. Older literature was not searched for as the standard of care in neonatal units has developed strongly in the last decade. Citations of eligible studies were checked for additional articles that might also be included.

\section{Study selection/eligibility criteria}

Studies were included if they: 1) reported on the presence of neonatal seizures in term (GA $>37$ weeks) infants; 2) excluded congenital brain abnormalities; 3 ) reported the etiology of the observed neonatal seizures; 4) the method of seizure diagnosis was described and consisted of at least a neurophysiology diagnosis either by full lead EEG or aEEG.; 5) reported on neurodevelopmental outcome of survivors with a minimum follow up age of 18 months using outcome and age appropriate neurodevelopmental testing or neurological examination, or on the presence or absence of post neonatal epilepsy at follow-up visits at any time. Both prospective and retrospective studies were eligible for inclusion.

Adverse outcome in survivors had to be defined by one or more of the following criteria: 1) the presence of cerebral palsy, either diagnosed by standardized clinical neurological examination or scored by the GMFCS gross motor functional classification system; 2) a test score of at least 2 standard deviations below the reference mean on the Bayley Scales of Infant Development (BSID) or the Griffith Mental Developmental Index or any other age appropriate neurodevelopmental test.

The presence of post neonatal epilepsy in survivors had to be clearly documented by full lead EEG diagnosis with either the need for continued antiepileptic drug (AED) use or the need for continued pediatric neurology outpatient visits. Studies only reporting clinical suspicion of seizures were not included for analysis.

Studies were excluded if: 1) they reported only experimental data; 2) they were performed in preterm infants; 3 ) they only reported clinical suspicion of seizures without neurophysiological diagnostics; 4) they reported on rare (either metabolic or other) syndromes; 5) they did not report developmental outcome data at $>18$ months of age or were not published in English. Also studies where it was not possible to extract data (e.g. original data not (clearly) described) or where it was not possible to make two by two tables were excluded from the analyses.

From the literature search a study selection was primarily made on the basis of Title, Abstract, Date and language. Full text articles were retrieved for final assessment. The same two reviewers who made the selection appraised the methodological quality (JL, TRH) and performed the data extraction independently. Disagreements were resolved by discussion until consensus was reached.

Studies were only included for further analyses if each major category (see also Additional file 2: patient selection; diagnosis of neonatal epilepsy; reference standard outcome and flow and timing) could be assessed.

\section{Methodological quality/critical appraisal}

Due to a lack of existing quality assessment tools for prognostic studies, we developed a tool based on the modified version of the QUADAS-2 assessment tool [13] to evaluate the methodological quality of included studies. This tool (see also Additional file 2) consisted of 4 main items to assess methodological quality: 1) the patient selection process; 2) diagnosis of main determinant (neonatal seizures); 3) independent assessment of the outcome and 4) flow and timing.

Each main item was scored either positive or negative for good methodological quality by assessing sub-items answering questions regarding patient selection; inappropriate exclusion; sample properties; bias; blinding; conduct and interpretation of diagnostic testing and main outcome; uniformity in outcome assessment and percentage of loss to follow -up (if known). The number of items to be scored with yes/no/unclear was 20 . 


\section{Data-extraction}

Data extraction was performed using a standardized data extraction form (Additional file 3). As etiology is thought to influence prognosis to a great extent, extracted data were first categorized into different etiological subgroups: HIE, stroke and cerebral hemorrhage. Next, these subgroups were categorized as newborns with or without neonatal seizures, defined as seizures in the first 28 days of life. Extracted data were entered in 2 by 2 tables.

Separate 2 by 2 tables were made for the 2 outcomes of interest; 1) adverse or normal neurodevelopmental outcome and 2) post neonatal epilepsy present or absent.

Further data extracted were: year of publication, first author, disease (stroke; asphyxia or hemorrhage), mode of seizure diagnosis, imaging modality, mortality, total number of newborns in cohort, number of newborns with neonatal seizures, number of newborns with adverse outcome or with favorable outcome, number of newborns with post-neonatal epilepsy, study-design: prospective or retrospective; single or multicenter.

\section{Data analysis}

All data were entered in Review Manager version 5.3. Copenhagen: The Nordic Cochrane Centre, The Cochrane Collaboration, 2012; http://community.cochrane.org/help/ tools-and-software/revman-5/revman-5-download). To calculate a pooled Relative Risk with 95\% confidence interval (CI) for adverse outcome or development of postnatal epilepsy in patients with or without neonatal seizures we performed a meta-analysis using a univariate modeling approach. In view of the observed heterogeneity, a random-effects model was used. Heterogeneity was explored by the test for heterogeneity $\left(\mathrm{I}^{2}\right)$, which can be interpreted as the proportion of total variation observed between the trials attributable to differences between the studies, rather than chance. An $\mathrm{I}^{2}$ of more than $40 \%$ was considered to represent a heterogeneous meta-analysis.

\section{Results}

\section{Eligible studies}

Our primary search yielded 1443 studies, of which 1387 did not fulfill inclusion criteria, three studies were not written in English and 5 were narrative reviews with theoretical background information on this subject.

A total of 48 articles were selected for full text reading. From these 48 articles 39 studies were excluded as data could not be reliably extracted, leaving 9 studies for the final analyses: 4 studies on stroke, [14-17], 4 studies on HIE, [18-21] and one study on cerebral hemorrhage [22].

Figure 1 demonstrates the inclusion flow chart, and the characteristics of the included studies are shown in Table 1. The methodological quality of each included study as scored by our assessment tool is reported in Table 2 .

\section{Analyses of pooled data}

For all cases with stroke or asphyxia combined the pooled risk ratio (RR) for adverse outcome when suffering neonatal seizures was 7.42 (3.84-14.34), $\mathrm{I}^{2}=0 \%$, $\mathrm{Chi}^{2}=1.47$.

In neonates with perinatal asphyxia suffering neonatal seizures the pooled RR for adverse outcome was 8.41 (4.07-17.39), $\mathrm{I}^{2}=0 \%, \mathrm{Chi}^{2}=0.84$.

In neonates with stroke suffering neonatal seizures the pooled RR for adverse outcome 4.95 (1.07-23.0), $\mathrm{I}^{2}=0 \%, \mathrm{Chi}^{2}=0.21$.

The pooled RR for development of late onset epilepsy could only be determined for infants suffering from stroke: 1.48 (0.82-2.68), $\mathrm{I}^{2}=0 \%, \mathrm{Chi}^{2}=2,75$.

For both the etiologies HIE and cerebral hemorrhage only one study could be included which evaluated the development of epilepsy in infants with and without neonatal seizures. In the study of Pisani and colleagues $(n=57)$ late onset epilepsy was found in three HIE patients of in total 18 newborns suffering neonatal seizures and in none of the patients without neonatal seizures $(n=39)$. In the study of Beslow and colleagues $(n=17)$, including patients with cerebral hemorrhage, late onset epilepsy was found in one of the patients suffering neonatal seizures $(n=12)$ and in none of the patients without neonatal seizures $(n=5)$.

\section{Discussion}

To our knowledge this is the first systematic review and meta-analysis on the prognostic value of neonatal seizures for long term outcome in term newborns suffering from either perinatal asphyxia, stroke or cerebral hemorrhage.

Newborns suffering from hypoxic or vascular brain injury and seizures in the neonatal period may have an increased risk of adverse neurodevelopmental outcome compared with newborns with the same brain injury without neonatal seizures. This is in accordance with previous findings in the literature [2-4, 7-9].

The results of this meta-analysis do not provide evidence for the theory that the seizures play a causative pathogenic role. However, this theory is supported by findings from experimental studies demonstrating multiple adverse cerebral effects of neonatal seizures. One of these adverse effects is microglia activation, as indicated by the morphologic changes and rapid production of pro inflammatory cytokines after the onset of acute seizures [23]. Microglia participate in "synaptic stripping" by detaching presynaptic terminals from neurons thereby altering brain structure and possibly brain function. Another seizure induced adverse effect on the brain 


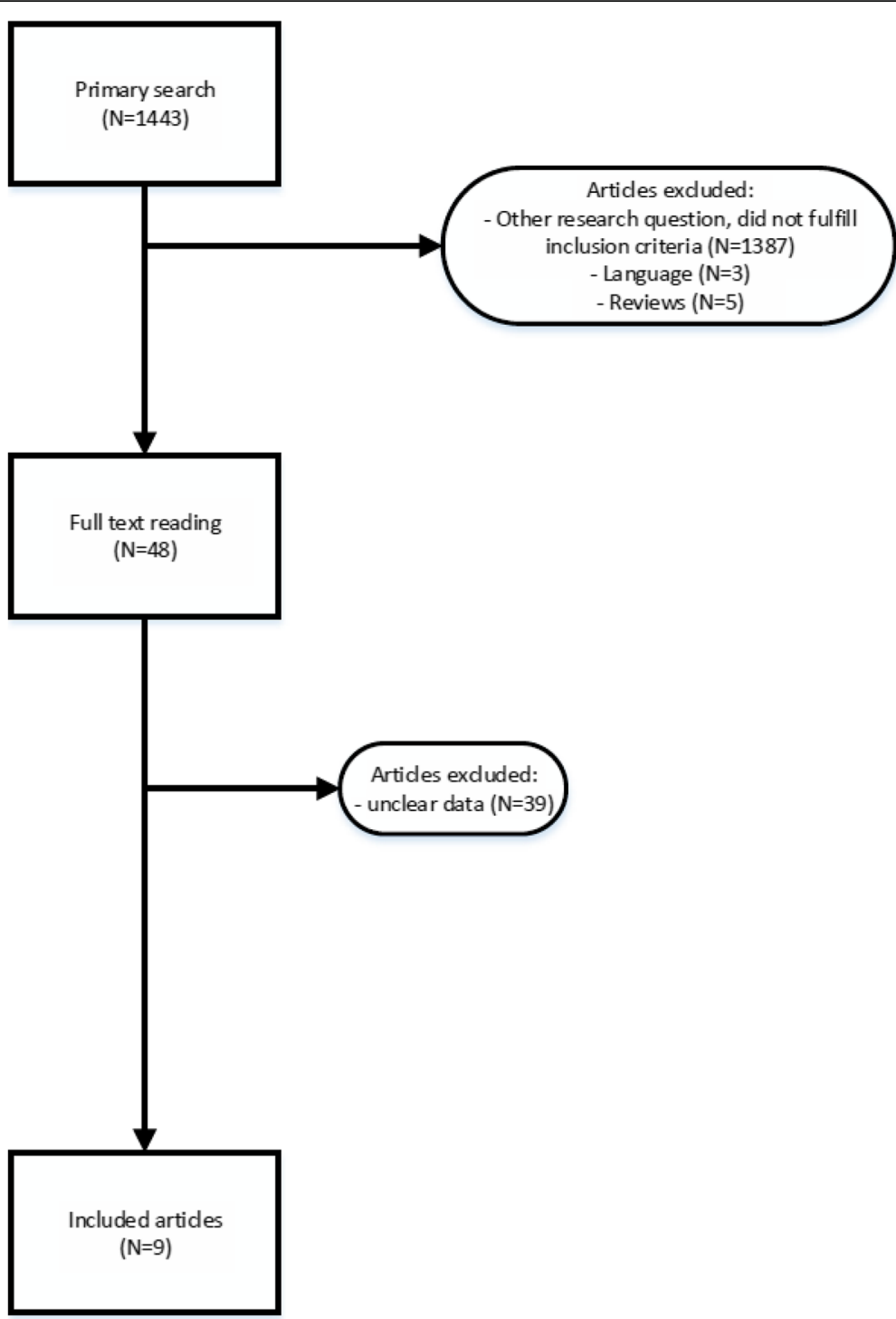

Fig. 1 Inclusion flow chart

development is a loss of subplate neurons, which are critical for the development of normal maturation of cortical networks [24].

The neonatal period is characterized by synaptic plasticity and learning. A major factor in this enhanced synaptic plasticity is the predominance of excitation over inhibition, which unfortunately also increases susceptibility to neonatal seizures. Seizure induced changes in these signaling pathways such as the GABA-ergic system, and changes in Glutamate receptors may result in long term impairments of brain function [5, 25].

These mechanisms may all influence long term outcome in newborns.

Most infants with neonatal seizures were treated with one or more anti-epileptic drugs (AEDs). There are reports that the use of (multiple) AEDs in the neonatal period is associated with adverse neurodevelopmental outcome [26, 27]. Obviously, this effect is confounded by indication and could not be well assessed on the basis of these data.

Although there is evidence that neonatal seizures influence both structure and function of the developing central nervous system, thereby possibly lowering the threshold for the development of epilepsy, we were not able to perform any meta-analysis on the risk for developing epilepsy in infancy. Only two studies, including small numbers of patients, reported on this outcome. The development of post neonatal epilepsy following neonatal seizures and brain damage may also be more difficult to predict as the location and severity of cortical 
Table 1 Characteristics of included articles

\begin{tabular}{|c|c|c|c|c|c|c|c|c|c|c|c|c|c|}
\hline Year & First author & Disease & $\begin{array}{l}\text { Disease } \\
\text { Diagnosis }\end{array}$ & N total & survivors & Died/ lost & NS & $\begin{array}{l}\text { Seizure } \\
\text { Diagnosis }\end{array}$ & Normal & Adverse & Epilepsy & Design $P / R$ & $S / M$ \\
\hline 2000 & C. Sreenan & Stroke & CT & 46 & 46 & $0 / 0$ & 42 & Clinical and EEG & 15 & 31 & 21 & $P$ & M \\
\hline 2007 & M.R. Golomb & Stroke & $\mathrm{CT} / \mathrm{MRI}$ & 64 & 61 & $0 / 3$ & 48 & EEG & - & - & 28 & $P$ & S \\
\hline 2008 & D.Ricci & Stroke & MRI & 31 & 28 & $1 / 2$ & 26 & EEG & 12 & 16 & 6 & $P$ & S \\
\hline 2010 & H.J. Lee & Stroke & MRI & $13^{b}$ & 7 & $0 / 0$ & 5 & EEG & 4 & 3 & 0 & $\mathrm{R}$ & M \\
\hline 2004 & S.P. Miller & Asphyxia & $\begin{array}{l}\text { ES } \\
\text { EEG }\end{array}$ & 68 & 60 & $8 / 0$ & 29 & Clinical mainly & 46 & 14 & - & $P$ & S \\
\hline 2009 & F. Pisani & Asphyxia & $\begin{array}{l}\text { Sarnat } \\
\text { Amiel -Tison } \\
\text { Cer. U.S. }\end{array}$ & $92^{\mathrm{a}}$ & 57 & $0 / 0$ & 18 & Video EEG & 47 & 10 & 3 & $\mathrm{R}$ & S \\
\hline 2009 & N. Al-Macki & Asphyxia & $\begin{array}{l}\text { Sarnat } \\
\text { EEG } \\
\text { MRI }\end{array}$ & 40 & 40 & $0 / 0$ & 33 & $\mathrm{EEG}$ & 17 & 23 & - & $\mathrm{R}$ & S \\
\hline 2009 & H.C. Glass & Asphyxia & MRI & 143 & 77 & $16 / 67$ & 25 & Video EEG & 60 & 17 & - & $P$ & S \\
\hline 2013 & L.A. Beslow & Hemorr. & CT MRI & $73^{c}$ & 20 & $1 / 0$ & 12 & Clinical and EEG & 16 & 1 & 1 & $P$ & M \\
\hline
\end{tabular}

Disease: brain injury type investigated in study; Disease Diagnose: diagnostic tool/measure/score assessing injury (Cer. US: cerebral ultrasound, MRI: magnetic resonance imaging): ES: encephalopathy score; $\mathrm{N}$ total: number of cases in study; Survivors: number of cases survived for follow up, NS: neonatal seizures number of cases; Adverse: adverse developmental outcome; Epilepsy: number of cases with late onset epilepsy; Design P/R: Prospective/Retrospective; S/M: Single-center/ Multi-center study. ${ }^{a} 92$ newborns with asphyxia, only 57 infants developed clinical HIE and were followed. ${ }^{b}$ total number included 13 but 6 preterm infants. ${ }^{c}$ total included 73 cases but only 20 perinatal term cases

damage may influence this. A recent literature study by Pisani et al. [28] demonstrated that around 18\% of newborns suffering from neonatal seizures (regardless of brain injury type) developed post-neonatal epilepsy. The onset of epilepsy was within the first year of life in almost $70 \%$ of cases. Uncertainty remains about the true incidence of post neonatal epilepsy after neonatal seizures due to the lack of well-designed population-based studies taking into account the type of brain injury.

We had hoped the large cohort studies on hypothermia would have contributed to answering our research question but unfortunately they did not fulfill our inclusion criteria as follow up criteria were not met and the incidence and treatment of seizures was not documented. Maybe an individual patient data analysis performed based on data from these large cohorts of affected newborns can answer our questions.

This study has some severe limitations that need to be discussed. First, the number of studies and patients included in this review was relatively small. Many studies had to be excluded because they included both newborns and older children or term and preterm newborns. Unfortunately, the data were not separately reported for the different age groups.

Second the mode of seizure - or epilepsy diagnosis was often not well defined and third the outcome in most studies was either not assessed in a blinded manner or outcome assessments were performed too early in life. This reduced the number of eligible studies greatly. Confirmation bias (a bias based on personal experience);

Table 2 Methodological quality of included studies

\begin{tabular}{|c|c|c|c|c|c|c|c|}
\hline Year & First author & Disease & $\begin{array}{l}1 \text { Selection } \\
6 \text { items }\end{array}$ & $\begin{array}{l}2 \text { Diagnosis } \\
3 \text { items }\end{array}$ & $\begin{array}{l}3 \text { Outcome } \\
5 \text { items }\end{array}$ & $\begin{array}{l}4 \text { Flow/Timing } \\
6 \text { items }\end{array}$ & $\begin{array}{l}\text { MQTotal } \\
20 \text { items } \\
\end{array}$ \\
\hline 2000 & C. Sreenan ${ }^{41}$ & Stroke & 5 & 3 & 3 & 5 & 16 \\
\hline 2007 & M.R. Golomb 22 & Stroke & 6 & 3 & 1 & 5 & 15 \\
\hline 2008 & D.Ricci ${ }^{37}$ & Stroke & 6 & 3 & 4 & 6 & 19 \\
\hline 2010 & H.J. Lee ${ }^{28}$ & Stroke & 6 & 3 & 3 & 6 & 18 \\
\hline 2004 & S.P. Miller ${ }^{31}$ & Asphyxia & 6 & 1 & 5 & 6 & 18 \\
\hline 2009 & F. Pisani $i^{35}$ & Asphyxia & 5 & 3 & 3 & 6 & 17 \\
\hline 2009 & N. Al-Macki ${ }^{5}$ & Asphyxia & 6 & 3 & 3 & 6 & 18 \\
\hline 2009 & H.C. Glass ${ }^{20}$ & Asphyxia & 6 & 3 & 5 & 4 & 18 \\
\hline 2013 & L.A. Beslow ${ }^{11}$ & Hemorrhage & 6 & 3 & 4 & 6 & 19 \\
\hline
\end{tabular}

Disease: brain injury type investigated in the study. The study score per main Quadas-2 item is demonstrated out of the maximum score per item. MQ total: total number of items scored positive for good methodological quality on Quadas-2 out of maximum of 20

Superscript numbers reflect the corresponding literature references 
unclear selection bias in the populations described; model under fitting (trying to explain a too complex disease entity or outcome by single determinants) and the presence of unknown cofounders may all have influenced our results.

However, taking all this into account, the data we reviewed give at least an indication that suffering neonatal seizures during neonatal stroke, brain hemorrhage or perinatal asphyxia may have an impact on outcome for the developing child.

For this reason, the results of our review need to be confirmed by large prospective cohort studies addressing these issues. Also a well-designed, multicenter registry prospectively collecting data on neonates with brain injury, including the presence or absence of neonatal seizures, is urgently needed. The diagnostic criteria and outcome parameters collected in such a database should be well-defined and uniformly assessed.

\section{Conclusions}

The presence of neonatal seizures combined with vascular or hypoxic brain injury in term newborns may have an impact on the neurodevelopmental outcome of affected newborns.

With the currently available data from the literature we could not determine the risk ratio of developing post-neonatal epilepsy following neonatal seizures and brain injury in term infants. One or more large prospective cohort studies are needed to confirm or refute these findings.

\section{Additional files}

Additional file 1: Search strategy. (DOCX $31 \mathrm{~kb}$ )

Additional file 2: Quadas Tool. (DOCX $21 \mathrm{~kb}$ )

Additional file 3: Data extraction form. (DOC $72 \mathrm{~kb}$ )

\section{Abbreviations}

AED: antiepileptic drug; BSID: Bailey States of Infant Development test; Cl: confidence Interval; GA: gestational age; GABA: Gamma Amino Butyric Acid; GMFS: Global Motor Functioning Scale; HIE: hypoxic ischemic encephalopathy; MOOSE: Meta-analysis of Observational Studies in Epidemiology; NICU: neonatal intensive care unit; PRISMA: Preferred Reporting Items for Systematic Reviews and Meta-Analyses; RR: risk ratio

\section{Availability of data and materials}

No individual patient data were collected or noted in any database. Data were collected form previously performed and published research as this was a literature study and Meta -analysis. Data were noted on assessment forms as described in the study methods. Additional information will be supplied by the authors upon request.

\section{Authors' contributions}

$\mathrm{TH}$ and $\mathrm{JHL}$ participated in the design of the study and performed the statistical analysis. AK participated in its design and coordination and helped to draft the manuscript. TH and JL performed the literature search, scored the articles on methodological quality and performed the data extraction. $\mathrm{JHL}$ also provided expert advice on methodological issues. All authors read and approved the final manuscript.
Ethics approval and consent to participate

Not applicable, this research concerned a literature search and Meta -analysis.

\section{Competing interests}

The authors declare that they have no competing interests.

\section{Publisher's Note}

Springer Nature remains neutral with regard to jurisdictional claims in published maps and institutional affiliations.

\section{Author details}

${ }^{1}$ Department of Neonatology, Emma Children's Hospital, Academic Medical Center, PO Box 22660, 1100, DD, Amsterdam, the Netherlands. ${ }^{2}$ Pediatric clinical Research Office, Emma Children's Hospital, Academic Medical Center, Amsterdam, The Netherlands.

Received: 25 January 2017 Accepted: 16 April 2018

Published online: 02 May 2018

\section{References}

1. Glass HC. Neonatal seizures: advances in mechanisms and management. Clin Perinatol. 2014;41:177-90.

2. Ramantani G. Neonatal epilepsy and underlying aetiology: to what extent do seizures and EEG abnormalities influence outcome? Epileptic Disord. 2013:15:365-75

3. Zupanc ML. Neonatal seizures. Pediatr Clin N Am. 2004;51:961-78. ix

4. van der Heide MJ, Roze E, van der Veere CN, ter Horst HJ, Brouwer OF, Bos AF. Long-term neurological outcome of term-born children treated with two or more anti-epileptic drugs during the neonatal period. Early Hum Dev. 2012;88:33-8.

5. Sanchez RM, Jensen FE. Maturational aspects of epilepsy mechanisms and consequences for the immature brain. Epilepsia. 2001:42:577-85.

6. Weeke LC, Groenendaal F, Toet MC, Benders MJ, Nievelstein RA, van Rooij $L G$, et al. The aetiology of neonatal seizures and the diagnostic contribution of neonatal cerebral magnetic resonance imaging. Dev Med Child Neurol. 2015:57:248-56.

7. Pisani F, Piccolo B, Cantalupo G, Copioli C, Fusco C, Pelosi A, et al. Neonatal seizures and postneonatal epilepsy: a 7-y follow-up study. Pediatr Res. 2012; 72:186-93.

8. Pisani F, Cerminara C, Fusco C, Sisti L. Neonatal status epilepticus vs recurrent neonatal seizures: clinical findings and outcome. Neurology. 2007;69:2177-85.

9. Nunes ML, Martins MP, Barea BM, Wainberg RC, Costa JC. Neurological outcome of newborns with neonatal seizures: a cohort study in a tertiary university hospital. Arq Neuropsiquiatr. 2008;66:168-74.

10. Kolk A, Ennok M, Laugesaar R, Kaldoja ML, Talvik T. Long-term cognitive outcomes after pediatric stroke. Pediatr Neurol. 2011:44:101-9.

11. Stroup DF, Berlin JA, Morton SC, Olkin I, Williamson GD, Rennie D, et al. Meta-analysis of observational studies in epidemiology: a proposal for reporting. Meta-analysis of observational studies in epidemiology (MOOSE) group. JAMA. 2000;283:2008-12.

12. Hutton B, Salanti G, Caldwell DM, Chaimani A, Schmid CH, Cameron C, et al. The PRISMA extension statement for reporting of systematic reviews incorporating network Meta-analyses of health care interventions: checklist and explanations. Ann Intern Med. 2015;162:777-84.

13. Whiting PF, Rutjes AW, Westwood ME, Mallett S, Deeks JJ, Reitsma JB, et al. QUADAS-2: a revised tool for the quality assessment of diagnostic accuracy studies. Ann Intern Med. 2011:155:529-36.

14. Golomb MR, Garg BP, Carvalho KS, Johnson CS, Williams LS. Perinatal stroke and the risk of developing childhood epilepsy. J Pediatr. 2007:151:409-13. e1-2

15. Lee HJ, Lim BC, Hwang H, Hong JS, Kim EK, Kim HS, et al. Clinical presentations and neurodevelopmental outcomes of perinatal stroke in preterm and term neonates: a case series. J Korean Med Sci. 2010;25: 888-94

16. Ricci D, Mercuri E, Barnett A, Rathbone R, Cota F, Haataja L, et al. Cognitive outcome at early school age in term-born children with perinatally acquired middle cerebral artery territory infarction. Stroke. 2008:39:403-10.

17. Sreenan C, Bhargava R, Robertson CM. Cerebral infarction in the term newborn: clinical presentation and long-term outcome. J Pediatr. 2000; 137:351-5. 
18. Al-Macki N, Miller SP, Hall N, Shevell M. The spectrum of abnormal neurologic outcomes subsequent to term intrapartum asphyxia. Pediatr Neurol. 2009;41:399-405.

19. Glass HC, Glidden D, Jeremy RJ, Barkovich AJ, Ferriero DM, Miller SP. Clinical neonatal seizures are independently associated with outcome in infants at risk for hypoxic-ischemic brain injury. J Pediatr. 2009;155:318-23.

20. Miller SP, Latal B, Clark H, Barnwell A, Glidden D, Barkovich AJ, et al. Clinical signs predict 30-month neurodevelopmental outcome after neonatal encephalopathy. Am J Obstet Gynecol. 2004;190:93-9.

21. Pisani F, Orsini M, Braibanti S, Copioli C, Sisti L, Turco EC. Development of epilepsy in newborns with moderate hypoxic-ischemic encephalopathy and neonatal seizures. Brain and Development. 2009;31:64-8.

22. Beslow LA, Abend NS, Gindville MC, Bastian RA, Licht DJ, Smith SE, et al. Pediatric intracerebral hemorrhage: acute symptomatic seizures and epilepsy. JAMA Neurol. 2013;70:448-54

23. Dommergues MA, Plaisant F, Verney C, Gressens P. Early microglial activation following neonatal excitotoxic brain damage in mice: a potential target for neuroprotection. Neuroscience. 2003;121:619-28.

24. Rakhade SN, Jensen FE. Epileptogenesis in the immature brain: emerging mechanisms. Nat Rev Neurol. 2009;5:380-91.

25. Sanchez RM, Koh S, Rio C, Wang C, Lamperti ED, Sharma D, et al. Decreased glutamate receptor 2 expression and enhanced epileptogenesis in immature rat hippocampus after perinatal hypoxia-induced seizures. J Neurosci. 2001;21: 8154-63.

26. Bittigau P, Sifringer M, Ikonomidou C. Antiepileptic drugs and apoptosis in the developing brain. Ann N Y Acad Sci. 2003:993:103-14. discussion 23-4

27. Bittigau P, Sifringer M, Genz K, Reith E, Pospischil D, Govindarajalu S, et al. Antiepileptic drugs and apoptotic neurodegeneration in the developing brain. Proc Natl Acad Sci U S A. 2002;99:15089-94.

28. Pisani F, Facini C, Pavlidis E, Spagnoli C, Boylan G. Epilepsy after neonatal seizures: literature review. Eur J Paediatr Neurol. 2015;19:6-14.

\section{Ready to submit your research? Choose BMC and benefit from:}

- fast, convenient online submission

- thorough peer review by experienced researchers in your field

- rapid publication on acceptance

- support for research data, including large and complex data types

- gold Open Access which fosters wider collaboration and increased citations

- maximum visibility for your research: over $100 \mathrm{M}$ website views per year

At BMC, research is always in progress.

Learn more biomedcentral.com/submissions 